\title{
Efficacy of Slow Reversal Hold and Isometrics in Improving Muscle Strength, Increasing Range of Motion, and Reducing Pain in Patients with Osteoarthritis of Knee
}

\author{
Harneet Arora \\ Department of Physical Therapy, University of Florida, Gainesville, FL, USA \\ Email: harora.phd@gmail.com
}

How to cite this paper: Arora, H. (2018) Efficacy of Slow Reversal Hold and Isometrics in Improving Muscle Strength, Increasing Range of Motion, and Reducing Pain in Patients with Osteoarthritis of Knee. Health, 10, 542-550.

https://doi.org/10.4236/health.2018.105043

Received: April 17, 2018

Accepted: May 13, 2018

Published: May 16, 2018

Copyright $\odot 2018$ by author and Scientific Research Publishing Inc. This work is licensed under the Creative Commons Attribution International License (CC BY 4.0).

http://creativecommons.org/licenses/by/4.0/

(c) (i) Open Access

\begin{abstract}
Osteoarthritis of knee is a common problem in the elderly population worldwide. Physical therapy has been shown to be useful in decreasing pain and increasing mobility in this population. The aim of this study was to study the effectiveness of slow reversal hold and isometric exercise techniques in reducing pain, increasing muscle strength, and increasing range of motion for knee flexion in patients with osteoarthritis of knee. The other aim of this study was to compare the relative effectiveness of these two interventions. 60 subjects participated in this study and were randomly assigned either to the slow reversal hold group $(\mathrm{n}=30)$ or, to the isometrics exercise group $(\mathrm{n}=30)$. All subjects performed their respective exercises for 3 weeks. Pain scores using the visual analog scale, muscle strength using manual muscle testing of quadriceps and hamstrings, and range of motion (ROM) for knee flexion using a goniometer were recorded both pre-treatment and post-treatment for both left and right knees. Both the interventions showed a significant decrease in pain scores, an increase in muscle strength, as well as an increase in the ROM. However, ROM was significantly increased in the slow reversal hold group as compared to the isometrics group in both knees. It was concluded that both exercise techniques could be useful in patients with osteoarthritis of knee for decreasing pain and increasing muscle strength. Slow reversal hold technique might be a better technique than isometrics for increasing ROM for knee flexion.
\end{abstract}

\section{Keywords}

Proprioceptive Neuromuscular Facilitation, Isometrics, Physical Therapy, Rehabilitation, Manual Muscle Testing, Pain, Knee Osteoarthritis 


\section{Introduction}

Osteoarthritis (OA) of knee is a common form of joint disorder, affecting the articular cartilage [1] [2]. OA could be caused by a damage to a joint cartilage. The risk factors for OA include obesity, genetics, previous fractures of the knee, and prolonged or excessive use of knee joints [2] [3]. OA results in symptoms such as a deep aching pain in the inner knee, stiffness, swelling, decreased range of motion (ROM), and muscle weakness [1] [3]. Usually, the treatment is symptomatic and involves the use of braces, non-steroidal anti-inflammatory drugs for pain relief, muscle relaxants, and exercise. Patients undergo a knee replacement if symptomatic treatment options have been ineffective [3].

Regular exercise is very important for controlling OA of knee successfully [4]. Stretching and strengthening exercises are useful for pain relief, increasing muscle strength, and increasing the ROM. Physical therapy aims to rehabilitate patients suffering from OA of knee by focusing on exercise plans and long-term rehabilitation goals. Several different types of exercises can be used for managing OA. Previous research has studied the positive effect of physical therapy in OA of knee [5] [6] [7] [8]. This study focused on two interventions-the slow reversal hold, which is a Proprioceptive Neuromuscular Facilitation (PNF) technique, isometric exercises, and their relative effectiveness. This comparison, to the best of my knowledge, has not been studied before. PNF has been proven to increase active and passive ROM of joints [9].

The aims of this study were: 1) to study the effectiveness of the slow reversal hold in treatment of osteoarthritis of knee, 2) to study the effectiveness of isometric exercises in treatment of osteoarthritis of knee, and 3) to study the comparison between slow reversal hold and isometrics in treatment of osteoarthritis of knee.

\section{Methods}

\subsection{Study Design}

This was a cross-sectional study with a sample size of 60 patients. Patients between ages of $50-60$ years (mean age of $54.52 \pm 3.33$ years) (males 27, females 33) with osteoarthritis of knee were selected. Convenience sampling was used to select the sample size. The study was undertaken at the Physiotherapy and Orthopedics departments of Guru Nanak Dev Hospital, Amritsar, India. Informed consent was obtained from the subjects. Inclusion criteria for selecting subjects were: 1) age between 50 - 60 years; 2) grade 2 osteoarthritis of knee, based on $\mathrm{X}$-rays and as referred by orthopedic doctor; 3 ) a manual muscle test grade of at least 3 for both quadriceps and hamstrings muscles. Subjects were excluded if they had: 1) visual deficits; 2) other musculoskeletal disorders like gout; 3) peripheral vascular diseases like deep vein thrombosis; 4) neurological disorders; 5) grade 3 or 4 osteoarthritis of knee; 6) knee replacement surgery; 7) knee deformities like genu valgum or genu varus; 8) sensory loss; 9) scars over knee; 10) uncooperative patients; 11) pyrexia or hypothermia; 12) extensor lag. The pur- 
pose of the study was explained to all the subjects who volunteered to participate and met the inclusion criteria. They were given information about the effects of both intervention techniques, prior to the treatment. An informed consent was obtained in their own understandable language.

\subsection{Protocol}

Subjects were randomly divided into two groups, each group containing $30 \mathrm{pa}$ tients. Two exercise interventions: slow reversal hold technique and isometrics of quadriceps and hamstrings, were performed on the subjects. In the slow reversal hold group, there were 17 females and 13 males and the isometrics group, consisted of 16 females and 14 males. Subjects were treated with either the slow reversal hold technique or isometrics of quadriceps and hamstrings, for duration of 3 weeks.

Pain was measured before and after the treatment by using visual analog scale (VAS). VAS is considered as the gold standard for measuring pain. This scale is easy to administer, and involves a $0-10 \mathrm{~cm}$ long line drawn on a piece of paper. At one end of the scale, it is marked as "no pain" and at the other end, it is marked as "extreme pain" [10]. Similarly, muscle strength was measured by using manual muscle testing (MMT) of quadriceps and hamstrings muscles before and after the treatment, in a standardized protocol as reported by Kendall [11]. For quadriceps MMT, the subjects were in a sitting position and were given consistent instructions of "Straighten your knee. Hold it. Don't let me bend it". For hamstrings MMT, subjects were in a prone position and were given instructions of "Bend your knee. Hold it. Don't let me straighten it". Range of motion (ROM) for knee flexion was measured before and after the treatment by using goniometer.

\subsection{Therapeutic Interventions}

Slow reversalhold: Slow reversal hold is defined as an "isotonic contraction of the agonist followed immediately by an isotonic contraction of the antagonist" [10]. The patient was laid down in a prone position, with the knee in extension and the hip in 0 degrees of rotation, abduction, and adduction. The pelvis was stabilized with one hand to prevent an anterior tilt. The contralateral extremity was kept flat on the table to provide additional pelvic stabilization. The patient was given standardized instructions of "Bend your knee. Hold it. Don't let me straighten it. Bend it more. Now, hold, then again bend it". Once the knee was fully flexed to the available range, the patient was asked to extend the knee. Resistance was given around the posterior surface of the leg just above the ankle. The patient was given instructions of "Straighten your knee. Hold it. Don't let me bend it. Straighten more. Now, hold, then again straighten it". This sequence was repeated 10 times.

Isometrics exercise: Isometric exercises are defined as exercises in which muscle length does not change during contraction [10]. For this exercise, the patient was 
in a supine position, with the knee in extension and the hip in 0 degrees of extension, abduction and adduction.

For isometrics exercise for quadriceps muscle: the patient was in a supine position and a rolled towel was placed under the exercising knee. The patient was asked to press the towel with the help of quadriceps muscle force, hold it for 10 secs and then relax. This was performed for 10 repetitions. The patient was given instructions of "Press the towel down, hold it now. Now, relax".

For isometrics exercise for hamstrings muscle: The patient was in a supine position and a rolled towel was placed under the heel which was being exercised. The patient was asked to press the towel with the force of the heel, hold it for 10 secs and then relax. This was performed for 10 repetitions. The patient was given instructions of "Press the towel down, hold it now. Now, relax".

\subsection{Statistical Analysis}

Non-parametric Mann-Whitney tests were used for comparing pre-treatment and post-treatment measurements as well as comparing slow reversal hold and isometrics techniques for all outcome measures. The significance value was set at 0.05 .

\section{Results}

\subsection{Comparison between Pre-Treatment and Post-Treatment in Osteoarthritis of Knee Patients}

Patients with OA of knee had significantly lower VAS scores post-treatment as compared to pre-treatment for both slow reversal hold and isometrics techniques for both knees (Figure 1). VAS scores of 6 to 9 were recorded pre-treatment, which significantly reduced to a range of 3 to 6 post-treatment for slow reversal hold and isometrics interventions in left knee. Similarly, in the right knee, patients had VAS scores ranging from 5 to 8 pre-treatment, which were significantly reduced to 2 to 5 post-treatment by slow reversal hold intervention. For isometrics intervention in the right knee, the VAS scores reduced from 6 to 8 (pre-treatment) to 3 to 6 (post-treatment). Patients also had significantly higher MMT grades for both quadriceps (Figure 2) and hamstrings (Figure 3) after treatment as compared to before the treatment for both left and right knees using both exercise interventions. Post-treatment, many patients had a grade 5 for MMT of quadriceps and hamstrings using these interventions. Similarly, patients had increased ROM for knee flexion in both knees for both slow reversal hold and isometrics, after the interventions as compared to the pre-treatment stage (Figure 4). Using slow reversal hold intervention, patients had mean ROM of 100.8 degrees in left knee and 101.6 degrees in right knee (pre-treatment), which significantly increased to 106.2 degrees in left knee and 107.0 degrees in right knee (post-treatment) respectively. Using isometrics intervention, mean ROM increased from 100.8 degrees to 103.9 degrees in the left knee and from 101.5 degrees to 104.7 degrees in the right knee. 
(a)

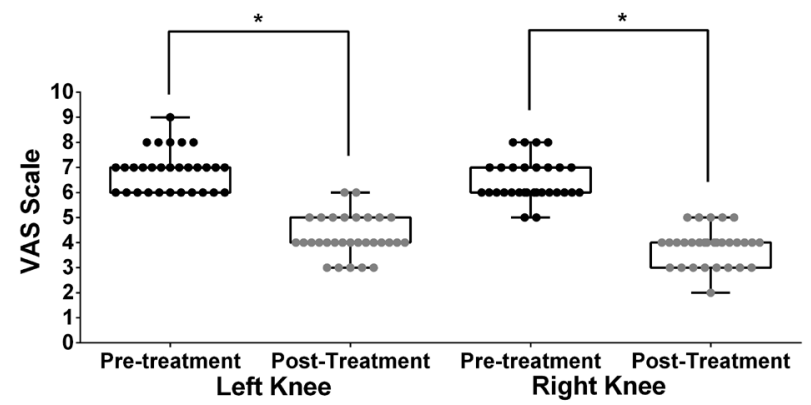

(b)

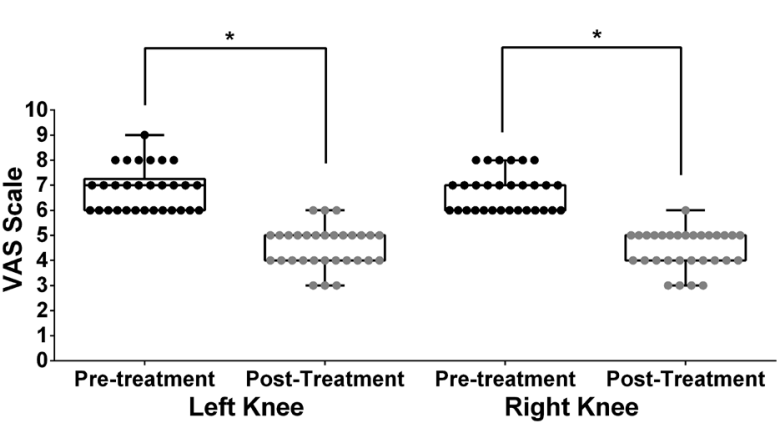

Figure 1. Comparison of visual analog scale between pre-treatment and post treatment for (a) slow reversal hold and (b) isometrics for both left and right knee in patients with osteoarthritis of knee. There was a significant difference between pre-treatment and post-treatment measurements for visual analog scores in both interventions-slow reversal hold and isometrics exercises in both knees. Data with range and standard error are shown, $\mathrm{p}<0.05$.

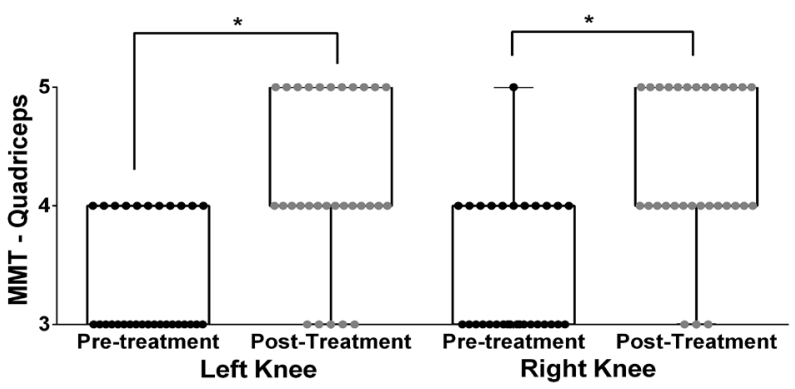

(b) Isometrics

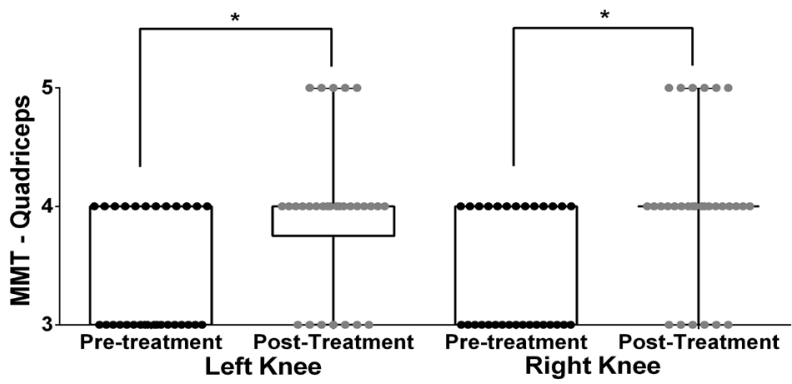

Figure 2. Comparison of manual muscle testing of quadriceps between pre-treatment and post-treatment for (a) slow reversal hold and (b) isometrics for both left and right knee in patients with osteoarthritis of knee. There was a significant difference between pre-treatment and post-treatment for manual muscle testing for quadriceps muscles for both interventions-slow reversal hold and isometrics exercises in both knees. Data with range and standard error are shown, $\mathrm{p}<0.05$.

(a)

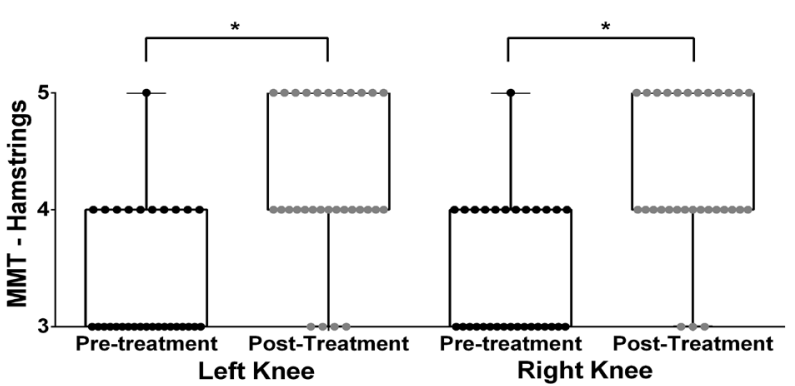

(b)

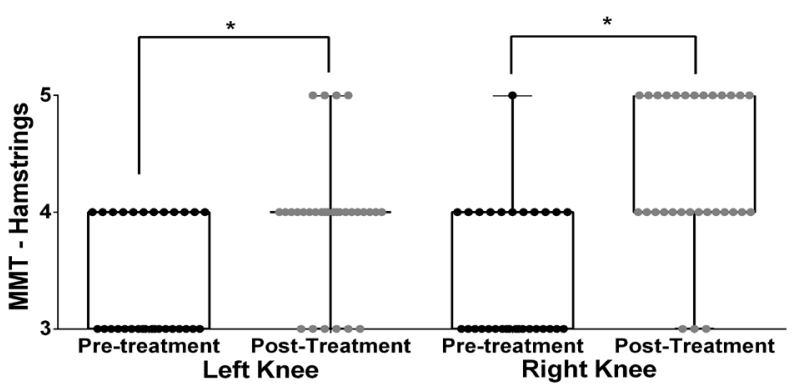

Figure 3. Comparison of manual muscle testing of hamstrings between pre-treatment and post-treatment for (a) slow reversal hold and (b) isometrics for both left and right knee in patients with osteoarthritis of knee. There was a significant difference between pre-treatment and post-treatment for manual muscle testing for hamstrings muscles for both interventions-slow reversal hold and isometrics exercises in both knees. Data with range and standard error are shown, $\mathrm{p}<0.05$.

\subsection{Comparison between Slow Reversal Hold and Isometrics in Osteoarthritis of Knee Patients}

On comparing both intervention types, it was observed that for the left knee, ROM for knee flexion was significantly higher in slow reversal hold (mean ROM 
(a)

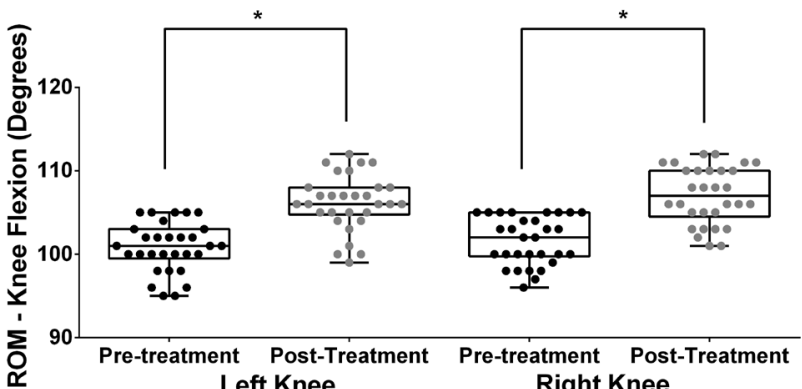

(b)

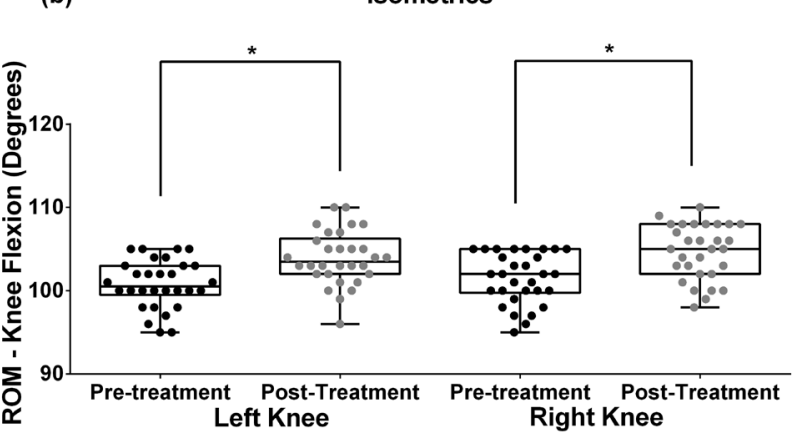

Figure 4. Comparison of range of motion for knee flexion between pre-treatment and post-treatment for (a) slow reversal hold and (b) isometrics for both left and right knee in patients with osteoarthritis of knee. There was a significant difference between pre-treatment and post-treatment for range of motion for knee flexion for both interventions-slow reversal hold and isometrics exercises in both knees. Data with range and standard error are shown, $\mathrm{p}<0.05$.

of 106.2 degrees) than in the isometrics intervention (mean ROM of 103.9 degrees) (Figure 5). For the right knee, patients in the slow reversal hold group had significantly lower VAS scores than patients in the isometrics group. Similarly, patients in the slow reversal group had significantly higher ROM for knee flexion (mean ROM of 107.0 degrees) than patients in the isometrics group for right knee (mean ROM of 104.7 degrees) (Figure 6).

\section{Discussion}

The primary purpose of this study was to see the effect of slow reversal hold and isometrics of knee interventions in osteoarthritis of knee. The second aim was to compare these two interventions for all the outcome measures evaluated in this study. It was observed that in patients with OA of knee, both slow reversal hold technique and isometrics of quadriceps and hamstrings were effective in terms of relieving pain, increasing muscle strength, and increasing ROM of knee flexion. Slow reversal hold performed better in increasing ROM for knee flexion in both knees.

Osteoarthritis of knee is a common problem, affecting many patients worldwide. In some cases, patients opt to undergo knee-replacement surgery to mitigate the increasing pain. Physical therapists play an important role in rehabilitation of these patients-both in pre-surgery and post-surgery phases. However, there has not been enough scientific evidence to determine the best intervention for treatment. This study showed that both slow reversal hold (PNF) technique and isometrics exercises would be beneficial in treating these patients, relieving their pain, increasing muscle strength, and increasing their ROM. This information would help physical therapists implement these techniques in a rehabilitation setting.

Slow reversal hold technique is a better intervention for increasing range of motion for knee flexion than isometrics. Isometrics exercises have been commonly used for OA of knee patients in a physical therapy setting. It is a good exercise that is easy to use for patients, and helps in relieving pain and strengthening 
(a)

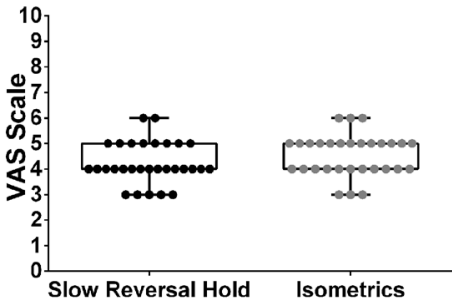

(c) MMT - Hams (Left Knee)

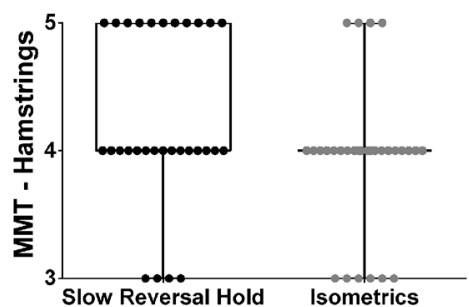

(b) MMT - Quads (Left Knee)

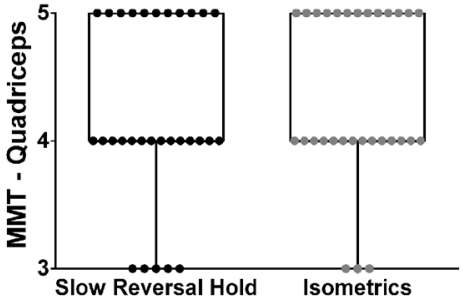

(d) ROM - Knee Flexion (Left Knee)

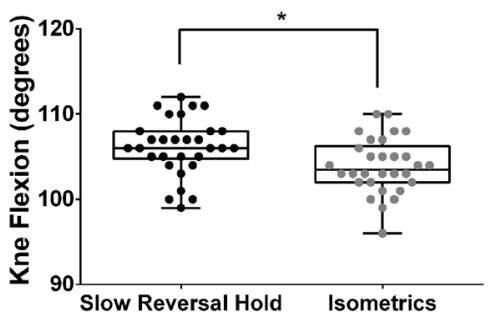

Figure 5. Comparison between slow reversal hold and isometrics for left knee for (a) visual analog scale; (b) manual muscle testing of quadriceps; (c) manual muscle testing of hamstrings, and (d) range of motion for knee flexion. There was no significant difference between the two interventions for any of the measures except range of motion for knee flexion. Slow reversal hold had significantly higher range of motion for knee flexion than isometrics for osteoarthritis of knee patients. Data with range and standard error are shown, $\mathrm{p}<0.05$.

muscles. However, slow reversal hold is better than isometrics for increasing ROM. One reason for this could be because slow reversal hold technique is similar to movements performed in daily living activities. Thus, it is a more active form of treatment. This information would help physical therapists to determine which type of exercise would be suitable for patients, depending on the treatment goals.

\section{Limitations}

This study had some limitations. Firstly, the study period was very short-3 weeks. The longer duration effects of both therapeutic interventions would have been useful for observing the bouts of remissions. Secondly, the effect of prescribed medications on the osteoarthritis of knee along with the exercises were not included in the study. Finally, it was hard to determine whether patients followed proper protocol when performing exercises at home.

\section{Conclusion}

Osteoarthritis is one of the most common problems faced by the elderly. Physical therapy could be of great use in rehabilitation of these patients. Both slow 
(a)

VAS (Right Knee)

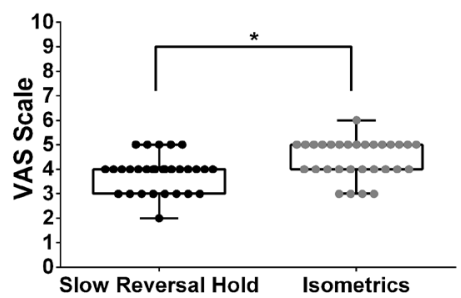

(c) MMT - Hams (Right Knee)

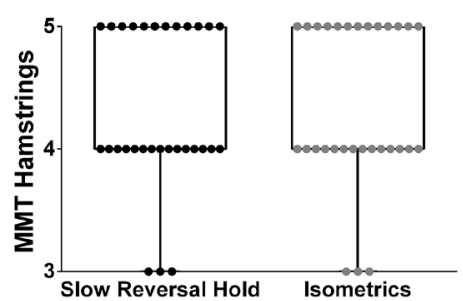

(b) MMT - Quads (Right Knee)

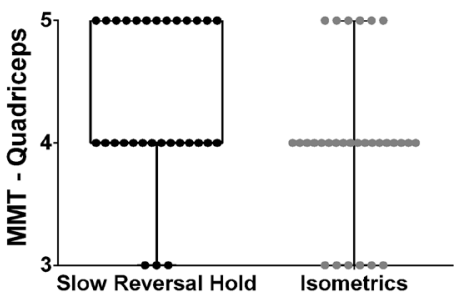

(d)ROM - Knee Flexion (Right Knee)

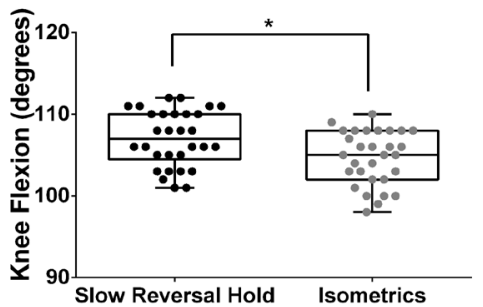

Figure 6. Comparison between slow reversal hold and isometrics for right knee for (a) visual analog scale, (b) manual muscle testing of quadriceps; (c) manual muscle testing of hamstrings, and (d) range of motion for knee flexion. There was no significant difference between the two interventions for any of the measures except visual analog scale and range of motion for knee flexion. Slow reversal hold had significantly higher range of motion for knee flexion and lower visual analog scores than isometrics for osteoarthritis of knee patients. Data with range and standard error are shown, $\mathrm{p}<0.05$.

reversal hold technique and isometrics are significantly effective in reducing pain, increasing muscle power, and increasing range of motion of knee flexion in patients with osteoarthritis of knee. Slow reversal hold could be more useful in increasing range of motion for knee flexion than isometrics exercise. This information would help physical therapists to make informed decisions about appropriate exercises for rehabilitation of osteoarthritis of knee patients.

\section{References}

[1] Khalaj, N., et al. (2014) Effect of Exercise and Gait Retraining on Knee Adduction Moment in People with Knee Osteoarthritis. Proceedings of the Institution of Mechanical Engineers Part H, 228, 190-199. https://doi.org/10.1177/0954411914521155

[2] Zhang, Y. and J.M. (2010) Jordan, Epidemiology of Osteoarthritis. Clinics in Geriatric Medicine, 26, 355-369. https://doi.org/10.1016/j.cger.2010.03.001

[3] CDC (2018) Osteoarthritis (OA). https://www.cdc.gov/arthritis/basics/osteoarthritis.htm

[4] Bosomworth, N.J. (2009) Exercise and Knee Osteoarthritis: Benefit or Hazard? Canadian Family Physician, 55, 871-878.

[5] Deyle, G.D., et al. (2000) Effectiveness of Manual Physical Therapy and Exercise in 
Osteoarthritis of the Knee. A Randomized, Controlled Trial. Annals of Internal Medicine, 132, 173-181. https://doi.org/10.7326/0003-4819-132-3-200002010-00002

[6] Bennell, K.L., et al. (2005) Efficacy of Physiotherapy Management of Knee Joint Osteoarthritis: A Randomised, Double Blind, Placebo Controlled Trial. Annals of the Rheumatic Diseases, 64, 906-912. https://doi.org/10.1136/ard.2004.026526

[7] Hinman, R.S., Heywood, S.E. and Day, A.R. (2007) Aquatic Physical Therapy for Hip and Knee Osteoarthritis: Results of a Single-Blind Randomized Controlled Trial. Physical Therapy, 87, 32-43. https://doi.org/10.2522/ptj.20060006

[8] Fransen, M., Crosbie, J. and Edmonds, J. (2001) Physical Therapy Is Effective for Patients with Osteoarthritis of the Knee: A Randomized Controlled Clinical Trial. The Journal of Rheumatology, 28, 156-164.

[9] Hindle, K.B., et al. (2012) Proprioceptive Neuromuscular Facilitation (PNF): Its Mechanisms and Effects on Range of Motion and Muscular Function. Journal of Human Kinetics, 31, 105-113. https://doi.org/10.2478/v10078-012-0011-y

[10] Abdel-Aziem, A.A., et al. (2018) Effect of a Physiotherapy Rehabilitation Program on Knee Osteoarthritis in Patients with Different Pain Intensities. Journal of Physical Therapy Science, 30, 307-312. https://doi.org/10.1589/jpts.30.307

[11] Kendall, F.P. (2005) Muscles: Testing and Function, with Posture and Pain (Kendall, Muscles). 5th, North American Edition, LWW, Philadelphia. 